\title{
A deep echelle survey and new analysis of diffuse interstellar bands ${ }^{\star}, \star \star$
}

\author{
S.Ó. Tuairisg ${ }^{1,2,3}$, J. Cami $^{4}$, B.H. Foing ${ }^{2}$, P. Sonnentrucker ${ }^{2}$, and P. Ehrenfreund ${ }^{1}$ \\ ${ }^{1}$ Leiden Observatory, P.O. Box 9513, 2300 RA Leiden, The Netherlands \\ 2 Solar System Division, ESA Space Science Department, ESTEC/SO, 2200 AG Noordwijk, The Netherlands \\ 3 Department of Physics, National University of Ireland Galway, Ireland \\ 4 Astronomical Institute "Anton Pannekoek", University of Amsterdam, Kruislaan 403, 1098 SJ Amsterdam, The Netherlands
}

Received October 30, 1998; accepted November 10, 1999

\begin{abstract}
We report a deep survey of diffuse interstellar bands (DIBs) between $3906 \AA$ and $6812 \AA$ under consistent observing conditions toward three very reddened and five unreddened stars. BD $+63^{\circ} 1964$ 's line-of-sight was shown to present exceptional DIB enhancement in number as well as in strength. The early spectral type of the star and the use of spectra of an unreddened comparison star of the same spectral type allowed to limit stellar line residuals. Using careful reduction and analysis methods we discovered 60 new DIBs which are confirmed in the reddened targets HD 183143 and $\mathrm{BD}+40^{\circ} 4220$. We detected 25 possible DIBs which still await further confirmation, but we did not detect or confirm 28 previously reported DIBs. The present survey with 226 confirmed DIBs, measured in three targets allows a detailed and homogeneous statistical analysis on the distribution of DIB widths and intensities.
\end{abstract}

Key words: ISM: general — ISM: molecules

\section{Introduction}

Superimposed on the extinction curve are a huge number of absorption lines, the diffuse interstellar bands (DIBs). The identification of the DIB carriers remains an important problem in astronomy. The current number of $\sim 200$ DIBs is still increasing, suggesting that more than 400 DIBs down to the confusion limit could be detected in the interstellar medium. The development of DIB research in recent years indicates that most DIB carriers could

Send offprint requests to: P. Ehrenfreund

* Based on observations at Observatoire Haute Provence with the spectrograph ELODIE.

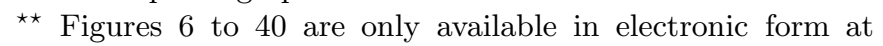
http://www.edpsciences.org be large carbon-bearing molecules which reside ubiquitously in the interstellar gas (see Herbig 1995 for a review, Salama et al. 1996). The first detection of substructures in the profile of several DIBs indicated the molecular nature of some DIB carriers (Sarre et al. 1995; Ehrenfreund \& Foing 1996). Foing \& Ehrenfreund $(1994,1997)$ observed two DIBs at 9577 and $9632 \AA$ as first evidence for $\mathrm{C}_{60}{ }^{+}$, the largest molecule ever detected in space. A survey of DIB correlations over $4000 \AA$ showed that most of the DIB carriers are undergoing photo-ionization and that all measured DIBs do originate from different carriers (Cami et al. 1997).

Recently many laboratories, theoreticians and observational astronomers showed combined efforts to solve the long-standing mystery of the DIBs and to identify their carriers. The spectra of $\mathrm{PAH}$ and fullerene cations, measured in a Neon matrix, carbon chains measured in the gas phase and theoretical calculations of the non-linear $\mathrm{H}_{2}$ DIB model have all shown some coincidences with some diffuse bands (see Salama et al. 1996; Foing \& Ehrenfreund 1997; Freivogel et al. 1994; Sorokin et al. 1996; Ubachs et al. 1997; Tulej et al. 1998).

Another approach to identify the DIB carriers is to study the complete DIB spectrum in different interstellar and circumstellar regions and to relate the line-of-sight conditions directly to the formation/evolution and destruction properties of DIB carrier molecules. Additional observations of spectral molecular features such as $\mathrm{CH}$, $\mathrm{CH}^{+}, \mathrm{CN}$ (as well as atomic lines CaI, CaII, NaI) reveal variations of physical parameters of the interstellar environment and can constrain the chemistry, ionization balance, metallicity and electron density in the circumstellar and interstellar environment. To define those parameters and to relate them to the DIB behaviour in the same region allows another view on the nature of the DIB carriers.

Observations show currently up to $\sim 200$ DIBs in dense and cold environments as well as in UV dominated 
regions. Their central wavelength is extremely constant. The band strength of the strongest DIBs (such as the 5780 and $5797 \AA$ DIBs, which are measured towards more than 200 sources) does not change by more than a factor $\sim 2$. The relative DIB strength, $W / E_{(B-V)}$, seems to reflect an interplay between ionization and recombination/destruction of the DIB carrier molecules. Even the high resolution profile of the $6613 \AA$ DIB, which shows a characteristic triple peak, displays only slight changes in different environments.

A new reference target for DIB studies was recently detected, which shows the strongest DIBs ever measured and allows to define the DIBs in several categories which respond in totally different ways to the local environment (Ehrenfreund et al. 1997). We discuss the optical survey towards the star $\mathrm{BD}+63^{\circ} 1964$ which led to the detection of 60 new DIBs. We present the complete DIB spectrum between 3906 and $6812 \AA$ of $\mathrm{BD}+63^{\circ} 1964$ and two other heavily reddened reference targets, HD 183143 and $\mathrm{BD}+40^{\circ} 4220$, and discuss the statistics of the confirmed DIBs.

\section{Previous DIB surveys}

In recent years the number of known DIBs has experienced considerable growth. This rapid increase is due to the improvement of CCDs and higher resolution instruments in the optical range. Herbig (1975) announced a total of 39 identified diffuse interstellar bands between 4400 and $6850 \AA$. In high signal to noise scans of reddened stars Herbig (1988) reported the discovery of 29 new lines between 6767 and $6862 \AA$. In a later publication Herbig \& Leka (1991) added 22 more DIBs to an ever-growing list. Most of these surveys concentrated on the star HD 183143. It was clear at this point that the main barriers in revealing more DIBs was instrumental. To date, the largest DIB survey was reported by Jenniskens \& Désert (1994). This survey included all the previously identified DIBs, and yet higher resolution than before resulted in the identification of more DIBs. The survey, from $3800 \AA$ to $8680 \AA$, revealed a total of 229 DIBs. This list included 153 certain DIBs as well as 76 probable and questionable DIBs. The survey covered a wider wavelength range than the present one of $\mathrm{BD}+63^{\circ} 1964$, but some regions of the spectrum were omitted in their data. A subsequent smaller survey, but using a resolution of 60000 concentrated on several different wavelength regions (Krelowski et al. 1995). These were mostly 80 $\mathrm{A}$ windows centred at $5790 \AA, 5915 \AA, 6010 \AA$, $6250 \AA, 6400 \AA$ and $6665 \AA$. This survey resulted in the tentative identification of 42 weak DIBs. These DIBs were labeled either as certain or as doubtful DIBs.

The total number of diffuse interstellar bands identified in the wavelength range $3800 \AA$ to $6815 \AA$ until now is 184,103 of these being classified as "certain" by
Jenniskens \& Désert (1994). What makes this present survey of these three reference targets both unique and necessary is: (i) the enhancement of narrow DIBs in $\mathrm{BD}+63^{\circ}$ 1964, mentioned below, enabling us to confirm many of the weaker DIBs already reported, as well as to discover many new DIBs. (ii) Never before has there been a survey of diffuse interstellar bands from $3980 \AA$ to $6820 \AA$ in one echelle spectrum. To cover a wide wavelength range it was necessary for previous surveys to take a number of different exposures of the same stars over a period of several months. This created numerous complications such as different seeing and telluric conditions, and calibration drifts, which do not arise in our data. We have analysed a consistent, deep exposure over the desired wavelength range of the star $\mathrm{BD}+63^{\circ} 1964$ which shows, to date, the strongest DIBs ever measured. (iii) We measured a number of unreddened targets to optimize instrumental, telluric and stellar line corrections. (iv) The detected DIB candidates have been checked for confirmation in deep spectra of two very reddened targets, HD 183143 and $\mathrm{BD}+40^{\circ}$ 4220, which have very different spectral types and rotation.

\section{Observations and data reduction}

\subsection{ELODIE spectrograph performances}

Observations were obtained on July 24th and November 8th, 1995 at the Observatoire de Haute Provence (OHP). We used the $1.93 \mathrm{~m}$ telescope, equipped with the spectrograph ELODIE, which is a fiber-fed echelle spectrograph, covering the wavelength range from 3906 to $6811 \AA$ with a resolution of $\sim 42000$ (Baranne et al. 1996). The fibres are POLYMICRO fibres with a diameter of $100 \mu \mathrm{m}$. The grating used is a $408 \times 102 \mathrm{~mm}$ echelle grating with 31 grooves $/ \mathrm{mm}$ and a $\theta=76^{\circ}$ blaze angle. The dispersion crossing is done with two optical components, a $40^{\circ}$ flint prism and a $8.63^{\circ}$ crown grism with 150 grooves $/ \mathrm{mm}$. The combination results in a constant interorder spacing over the 67 spectral orders. The detector is a back-illuminated Tk1024 CCD with $24 \mu \mathrm{m}$ pixels, cooled to $183 \mathrm{~K}$. At a readout of $100 \mu \mathrm{s} /$ pixel the typical readout noise is $8.5 \mathrm{e}^{-}$. The CCD has a linear response up to $100000 \mathrm{e}^{-}$.

The preliminary reduction of the ELODIE data was performed on-line at the observatory. The normal CCD reduction procedure was carried out, incorporating the correction of bad-pixels and cosmic rays, and the subtraction of the bias and dark current. No systematic post-reduction deglitching was applied except for very obvious spikes.

\subsection{The program stars and optimal reduction}

In addition to $\mathrm{BD}+63^{\circ} 1964$, observations of three different standard stars were performed during the same night. Table 1 summarises the physical and observational characteristics of all the stars used in this survey. HD 37128, 
Table 1. Physical and observational parameters for the program stars used in the DIB survey. The radial and rotational velocities were determined from the spectra

\begin{tabular}{|l||cccccccc|}
\hline Target & BD $+63^{\circ}$ & HD & BD $+40^{\circ}$ & HD & HD & HD & HD & HD \\
& 1964 & 183143 & 4220 & 32630 & 37128 & 205021 & 164353 & 188209 \\
\hline \hline$V$ magnitude & 8.6 & 6.86 & 9.05 & 3.20 & 1.70 & 3.19 & 3.97 & 5.62 \\
Spectral Type & B0 II & B7Iae & O7e & B3 V & B0 I & B1 IV & B5Ib & O9.5Ib \\
$E_{(B-V)}$ & 1.01 & 1.28 & 2 & 0.02 & 0.08 & 0 & 0.12 & 0.15 \\
$v \sin i\left(\mathrm{~km} \mathrm{~s}^{-1}\right)$ & 84 & 60 & $>300$ & 132 & 87 & 28 & 5 & 77 \\
$v_{\text {rad }}\left(\mathrm{km} \mathrm{s}^{-1}\right)$ & -91 & +34 & $\sim-140$ & +7 & +26 & -8 & +20 & +14 \\
Observed at: & & & & & & & & \\
$S / N$ & 205 & 200 & 88 & 274 & 387 & 390 & 228 & 214 \\
Airmass $(\operatorname{Sec} z)$ & 1.07 & 1.11 & 1.03 & 1.46 & 1.70 & 1.12 & 1.36 & 1.03 \\
\hline
\end{tabular}

with its comparable rotation and spectral type to $\mathrm{BD}+63^{\circ}$ 1964 was used as the main stellar standard. HD 205021, a slow rotator, further enabled us to control the identification of stellar lines in $\mathrm{BD}+63^{\circ} 1964$. HD 32630 was used as a telluric standard.

$\mathrm{BD}+63^{\circ} 1964$, is a B0 II star and has therefore relatively few stellar lines. Nevertheless the spectrum has, especially towards the blue, some prominent stellar lines which hamper the detection and measurement of possible new DIBs. Few stellar lines appear above $4800 \AA$ where most of the diffuse interstellar bands are concentrated. To distinguish DIBs from stellar lines the spectrum of $\mathrm{BD}+63^{\circ} 1964$ was divided by the stellar standard HD 37128 , which is a very good match in spectral type and rotational velocity. The resulting spectrum is characterised mostly by interstellar features, with a few residual stellar lines.

Telluric contamination limits the detection and measurement of DIBs and affects a significant fraction of our total wavelength range. The contamination is due to oxygen lines, mostly around the diffuse band at $6284 \AA$ and water lines above $5800 \AA$. Although the oxygen column density remains stable throughout the observations, the $\mathrm{H}_{2} \mathrm{O}$ column density can vary slightly between exposures. For telluric correction the bright star HD 32630 was chosen. To remove the atmospheric contamination the relation below is used:

$I_{\mathrm{TC}}=\frac{I}{I_{\mathrm{t}}^{\frac{z}{z_{t}}}}$

$I$ and $I_{\mathrm{t}}$ are the object spectrum and the telluric standard spectrum respectively. $\frac{z}{z_{t}}$ is the ratio of the respective airmasses. The inaccuracy in airmass estimations usually requires some iterations to obtain the true ratio. In removing stellar lines from regions contaminated by telluric absorption, both $\mathrm{BD}+63^{\circ} 1964$ and HD 37128 were first divided by HD 32630 .

The heavily reddened star $\mathrm{BD}+40^{\circ} 4220\left(E_{(B-V)}=2\right)$ and HD $183143\left(E_{(B-V)}=1.28\right)$, up to now the reference star for studies of DIB behaviour, were used to add confirmation to the newly detected DIBs in this present survey. HD 183143, a B7 star, has been used in previous DIB surveys (Herbig 1975; Jenniskens \& Désert 1994; Herbig

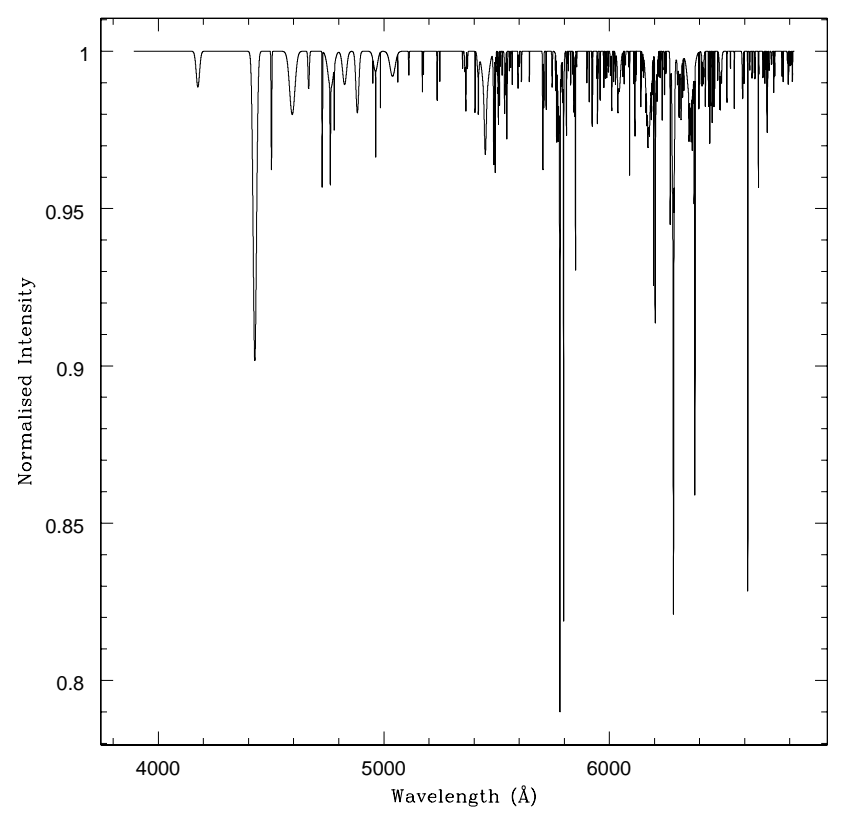

Fig. 1. A synthetic spectrum of all 226 DIBs confirmed towards $\mathrm{BD}+63^{\circ} 1964$ between $3906 \AA$ and $6812 \AA$

1995). $\mathrm{BD}+40^{\circ} 4220$ is a hot star (spectral type O7) with a fast rotation which is ideal for detecting weak DIBs, especially in the visible and infrared. HD 164353 was used to identify stellar lines and remove telluric contamination in HD 183143, and HD 188209 was used as BD+40 4220's telluric standard. Due to slight reddening in the standards HD 164535 and HD 188209, the large DIBs at $6284 \AA$ and $6283 \AA$ in these stars incurred an additional error of $\sim 10 \%$. See Fig. 40 for samples of corrected and uncorrected telluric contamination in the target stars.

\section{DIB measurement methods}

Central wavelengths, equivalent widths and full widths at half maximum were measured in the spectra of the three reddened stars $\left(\mathrm{BD}+63^{\circ} 1964, \mathrm{BD}+40^{\circ} 4220\right.$, and HD 183143) using the IRAF (Image Reduction and 


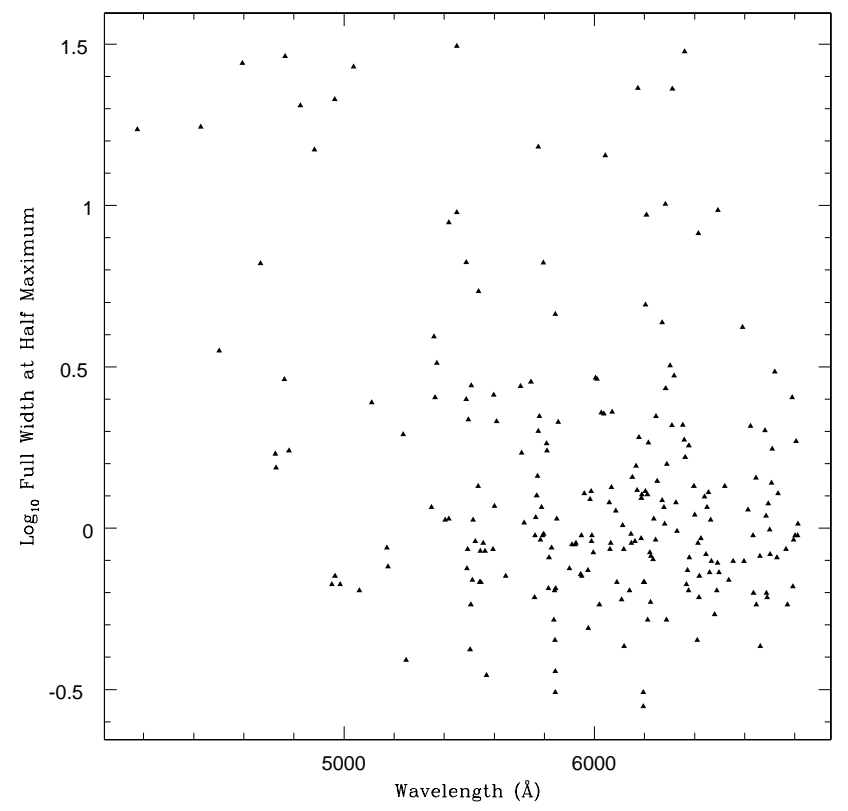

Fig. 2. A plot of $\log _{10}(F W H M)$ against wavelength for the DIBs measured toward $\mathrm{BD}+63^{\circ} 1964$. A dominant population of DIB widths lies between 0.6 and $1.4 \AA$, more abundant towards the red and clustered around some ranges. A second population of medium-broad DIBs is apparent with $F W H M$ between 1.4 and $3.2 \AA$. It also illustrates the clustering of some DIBs around "magic" wavelengths, at 550, 580 and $630 \mathrm{~nm}$

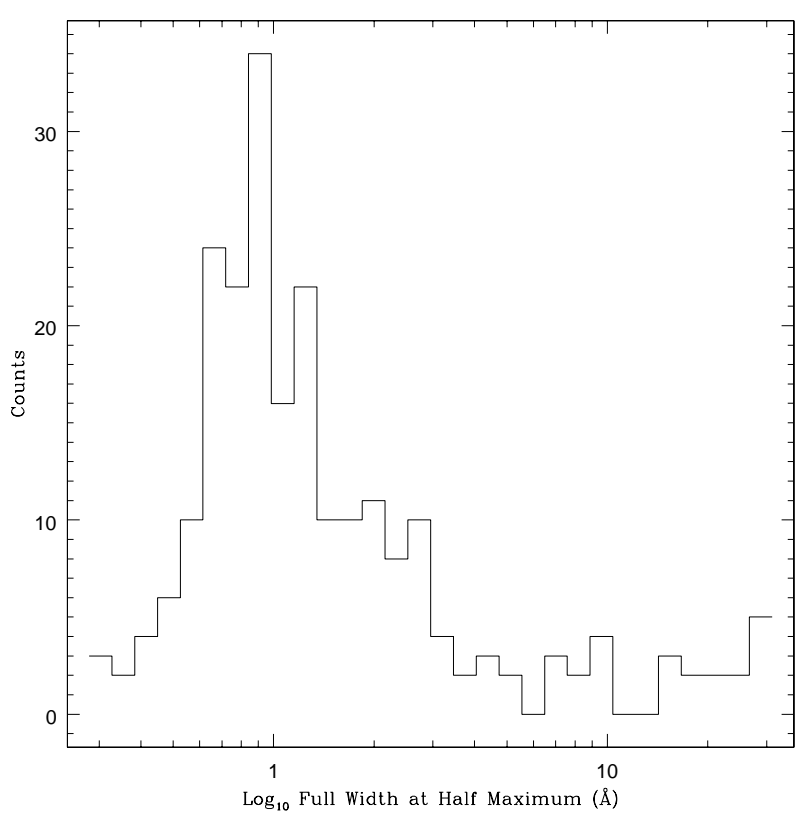

Fig. 3. Histogram of Full Widths of DIBs in BD $+63^{\circ}$ 1964. This plot illustrates the three populations of DIBs discussed in the text: narrow DIBs, medium broad DIBs and broad DIBs

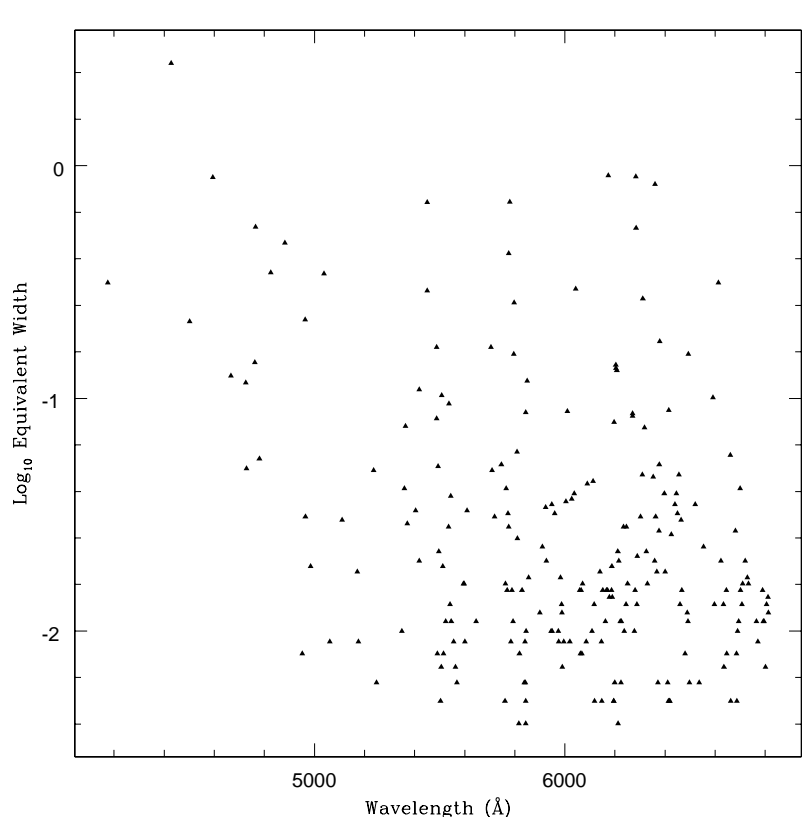

Fig. 4. A plot of $\log _{10}(E W)$ against wavelength for the DIBs measured toward $\mathrm{BD}+63^{\circ}$ 1964. This plot illustrates the increased number of DIBs detected for a given equivalent width towards the red, and the clustering of DIBs around 550, 580, and $630 \mathrm{~nm}$

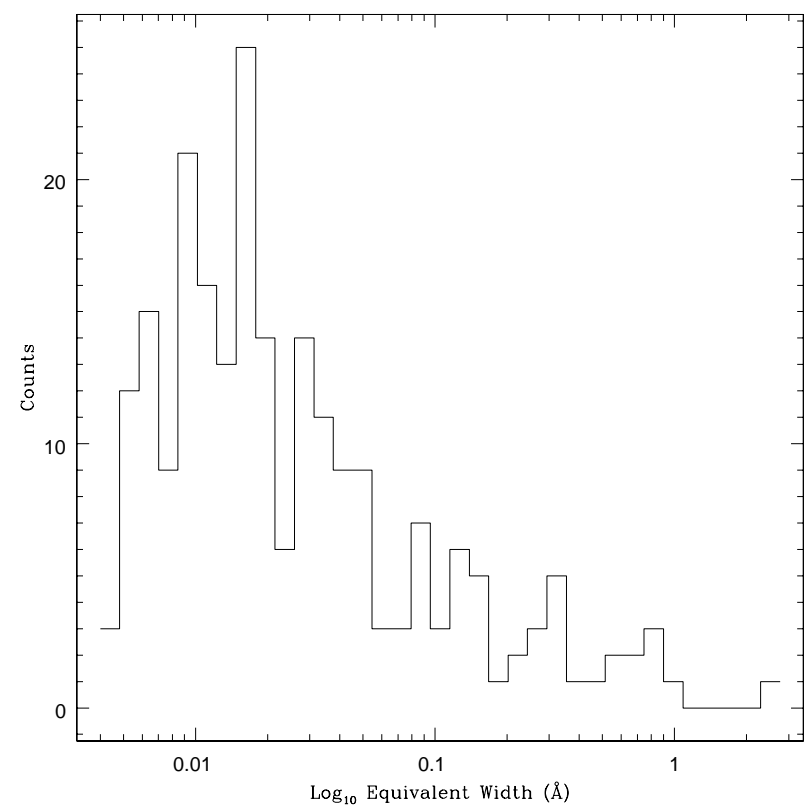

Fig. 5. Histogram of Equivalent Widths of DIBs in $\mathrm{BD}+63^{\circ}$ 1964. The cut off at $\sim 5 \mathrm{~m} \AA$ corresponds to the sensitivity limit for confident detection 
Analysis Facility) software package. Equivalent widths were obtained by integration of the pixel intensities within the region:

$W=\sum_{i}\left(1-\frac{I_{i}}{C_{i}}\right)$

where $I_{i}$ is the intensity at pixel $i$ and $C_{i}$ is the continuum level at pixel $i$. The central wavelength was estimated by calculating:

$\lambda_{\mathrm{c}}=\frac{\sum_{i} \lambda_{i}\left(I_{i}-C_{i}\right)^{\frac{3}{2}}}{\sum_{i}\left(I_{i}-C_{i}\right)^{\frac{3}{2}}}$

where $\lambda_{i}$ is the wavelength of pixel $i$. Alternatively a Gaussian profile can be fitted to the feature, resulting in the additional estimation of the full width at half maximum. Accurately defining the continuum level, which is especially important in measuring medium-broad to broad DIBs, involves fitting a polynomial to the continuum and dividing it out of the spectrum.

Error estimates, using a model based on the Poisson statistics of the data, were also obtained using the IRAF package. An error estimate model is fit to the data using as input a constant $\sigma$ estimated from the statistics of the spectrum. A number of simulations are created in which random Gaussian noise is added to the noise-free spectrum using the pixel sigmas from the noise model. The model fitting is done for each simulation and the absolute deviation of each fitted parameter to the noise-free model parameter is recorded. The error estimate for each parameter is then calculated as that deviation, inside which contains $68.3 \%(1 \sigma)$ of the parameter estimates.

The wavelengths of absorption bands of certain known molecular, atomic and ionic species (Ca II, Ca I, CH and $\left.\mathrm{CH}^{+}\right)$residing in the line of sight towards $\mathrm{BD}+63^{\circ} 1964$ were measured and the shift in wavelength between those lines and their values recorded in the laboratory was calculated. The sodium lines at $5890 \AA$ and $5895 \AA$ were saturated and so were not included in these calculations. Under ideal conditions the velocity shift would be identical for all lines. However the presence of at least two main clouds of similar column densities and of different velocities (the velocity difference between two substructures in the molecular absorption line was measured to be $\sim 8 \mathrm{~km} \mathrm{~s}^{-1}$ ) in the line of sight of $\mathrm{BD}+63^{\circ} 1964$ and the resulting twocomponent structure of the interstellar lines limited the estimation of the correct wavelength to an accuracy of $\sim 0.05 \AA$. The average central wavelengths of the interstellar lines were measured and an average of the velocity shift of $19.5 \pm 0.5 \mathrm{~km} \mathrm{~s}^{-1}$ was obtained based upon these measurements. The DIBs' measured wavelengths were then shifted by this amount to provide reliable laboratory wavelengths.

\section{Selection criteria for new DIBs}

Despite the high quality of $\mathrm{BD}+63^{\circ} 1964$ 's spectrum, the presence of weak spurious absorption features, including uncorrected or poorly corrected bad pixels and cosmic rays, atmospheric residuals left after the telluric correction and mismatches in stellar abundances between $\mathrm{BD}+63^{\circ}$ 1964 and HD 37128, results in the need for strong and consistent criteria for the positive identification of new DIBs. We checked that new DIBs could not be ascribed to such spurious features.

\subsection{The standard stars and synthetic stellar spectrum}

Although standard stars HD 37128 and HD 205021 were used to identify and eliminate stellar lines from our target spectrum, we have additionally produced a synthetic spectrum using the TLUSTY and SYNSPEC software (a stellar atmospheres model and emergent line synthesis package) to confirm the DIB identification.

The synthetic stellar spectrum together with the spectra of the standard stars HD 205021 and HD 37128 allowed the identification of stellar lines to very good accuracy in the star $\mathrm{BD}+63^{\circ} 1964$. Some residual absorption in the divided spectrum which coincided exactly in wavelength with strong or moderate stellar lines together with a band width comparable to the rotational velocity of $\mathrm{BD}+63^{\circ}$ 1964 were ascribed to slight mismatches in stellar abundances between HD 37128 and $\mathrm{BD}+63^{\circ} 1964$ and thus were not due to bona fide DIBs in the line-of-sight.

\subsection{The presence of new DIBs in other reference targets}

The most important test of new DIBs are their occurence in other well known reddened stars. The large number of DIBs detected toward BD+63 1964 may be exceptional and some DIBs might possibly only be observed in such hot and heavily reddened targets. Nevertheless the considerable strength of several DIBs suggested they might be visible in other stars. As previously mentioned HD 183143 and $\mathrm{BD}+40^{\circ} 4220$ were chosen as our DIB reference targets. The latter was selected due to its very reddened lineof-sight, and HD 183143 is a well known DIB source and a main target of the DIB survey by Jenniskens \& Désert (1994) and Herbig (1995). HD 183143 (spectral type B7 I) suffers from some stellar line contamination and $\mathrm{BD}+40^{\circ}$ 4220 (spectral type O7) has a faint visible magnitude of 9.05. For $\mathrm{BD}+40^{\circ} 4220$ we could therefore obtain a spectrum with a signal-to-noise ratio of only 88 in $V$ but rising to 170 in the red.

All new DIB candidates were cross checked against these spectra and, after eliminating the possibility of stellar line contamination, 60 of these DIB candidates were confirmed in the spectrum of either HD 183143 or $\mathrm{BD}+40^{\circ}$ 4220. Only those DIBs visible in either BD $+40^{\circ} 4220$ or HD 183143 along with $\mathrm{BD}+63^{\circ} 1964$ were included in Table 4. 


\section{Results}

\subsection{Spectral displays and table}

Figures 6 to 39 display the ELODIE echelle spectral orders of $\mathrm{BD}+63^{\circ} 1964, \mathrm{BD}+40^{\circ} 4220$ and $\mathrm{HD} 183143$ over the wavelength range $3906-6812 \AA$. BD $+63^{\circ} 1964$ 's associated standard stars, HD 205021 and HD 37128, are also shown for the identification of stellar lines. BD $+63^{\circ} 1964$ 's telluric standard, HD 32630, is shown in a separate box. Also plotted is the spectrum of $\mathrm{BD}+63^{\circ} 1964$ corrected for stellar lines (divided by HD 37128). A synthetic spectrum of all DIBs seen in this survey of $\mathrm{BD}+63^{\circ} 1964$ is also included to easily identify confirmed DIBs. When necessary, a telluric correction was applied. This is indicated in the figure caption. Table 4 lists all the diffuse interstellar bands positively identified toward $\mathrm{BD}+63^{\circ} 1964$.

\subsection{New DIBs}

The 60 new DIBs discovered in $\mathrm{BD}+63^{\circ} 1964$ do not seem to adhere to any general pattern. They are spread throughout the spectrum, although occasionally clusters of new DIBs arise, and a slight bias can be seen towards the red end of the spectrum. The wavelength range between $6140 \AA$ and $6200 \AA$ is an example of a region with many new weak DIBs blended with the very broad $6173 \AA$ DIB. The vast majority of the new DIBs are weak, narrow features, although some medium-broad DIBs are also reported. This is probably because the ELODIE spectrograph is not suited for the detection of very broad DIBs due to each order's relatively narrow wavelength coverage and the difficulty in merging adjacent orders. Few new DIBs are seen at shorter wavelengths. There are three reasons that could account for this:

- The spectrograph is not as sensitive at shorter wavelengths, where the reddened target is fainter. The noise level is much higher, especially at the beginning of each order;

- The blue range is also dominated by stellar lines, which does not ease a DIB search in that region;

- There are fewer DIBs at a given equivalent width in this wavelength region. DIBs are undoubtedly concentrated towards the red end of the spectrum (this is discussed further in Sect. 7).

\subsection{Undetected DIBs mentioned in previous surveys}

Some DIBs previously identified in other surveys were not included in the list of DIBs identified in BD+63 1964 's spectrum. Some appeared to be completely absent, or not rising above the noise level of the spectrum. Others were so weak that, taking into account flat-fielding deviations and other spurious absorptions, the absolute confirmation
Table 2. Previously reported Diffuse Interstellar Bands that could not be detected or reliably measured in $\mathrm{BD}+63^{\circ}$ 1964 and which are not included in the present survey of DIBs. "+" and "o" denotes the classification of certain and probable DIBs according to Jenniskens \& Désert (1994)."?" denotes Krelowski's "doubtful" DIBs. The second column lists the DIB central wavelength quoted by the references given. The next two Col. contain data on these DIBs quoted from measurements by Jenniskens at http://wwwspace.arc.nasa.gov $/{ }^{\sim}$ leonid/DIBcatalog.html. The fourth column $(E W)$ shows upper limits as measured in our spectrum of $\mathrm{BD}+63^{\circ} 1964$ to be compared with Col. 2. See Table 4 for Reference codes

\begin{tabular}{|ccrrcll|}
\hline & $\begin{array}{c}\lambda_{\mathrm{c}} \\
(\AA)\end{array}$ & $\begin{array}{r}\text { E }(B-V) \\
(\mathrm{m} \AA)\end{array}$ & $\begin{array}{r}F W \\
(\AA)\end{array}$ & $\begin{array}{c}E W \\
(\mathrm{~m} \AA)\end{array}$ & Comments & Ref. \\
\hline+ & 4066.0 & 283 & 15 &.. & Stellar? & He95 \\
+ & 4880.4 & 22 & 1.35 & $<8$ & Weak & JD94 \\
o & 5845.4 & 4 & 0.7 &.. & Blend DIB & J96 \\
o & 5847.7 & 2 & 0.61 &.. & Absent & J96 \\
+ & 5850.2 & 6 & 1.5 &.. & Blend DIB & J96 \\
o & 5902.8 & 7 & 1.2 & $<6$ & Absent & K95 \\
+ & 5904.6 & 9 & 0.17 & $<6$ & Absent & K95 \\
o & 5908.3 & 3 & 0.6 & $<8$ & Weak & K95 \\
o & 5927.5 & 11 & 0.7 & $<6$ & Very Weak & K95 \\
o & 5929.6 & 7 & 0.6 &.. & Absent & K95 \\
$?$ & 5941.7 & - & - & $<6$ & Absent & K95 \\
$?$ & 5989.4 & - & - & 9 & Probable & K95 \\
$?$ & 5999.8 & 6 & 0.6 & $<8$ & Weak & K95 \\
o & 6032.9 & 5 & 0.6 &.. & Absent & K95 \\
+ & 6212.7 & 14 & 1.23 &.. & Absent & Ch85 \\
$?$ & 6238.8 & - & - & $<6$ & Absent & K95 \\
o & 6321.5 & 16 & 1.45 & $<6$ & Absent & JD94 \\
+ & 6451.6 & 403 & 25.4 &.. & Absent & JD94 \\
o & 6494.9 & 23 & 1.39 & $<6$ & Absent & JD94 \\
+ & 6532.1 & 664 & 17.2 &.. & too broad & JD94 \\
$?$ & 6635.3 & - & - & $<8$ & Weak & K95 \\
$?$ & 6649.8 & - & - & $<8$ & Very Weak & K95 \\
$?$ & 6693.3 & 5 & 0.8 & $<6$ & Blend DIB & K95 \\
$?$ & 6696.8 & - & - & $<6$ & Absent & K95 \\
+ & 6741.0 & 13 & 0.97 &.. & too broad & He75 \\
o & 6767.6 & 8 & 0.7 & $<8$ & Weak & He88 \\
o & 6768.6 & 5 & 0.7 & $<6$ & Absent & He95 \\
o & 6779.0 & 3 & 0.54 & $<8$ & Weak & He95 \\
\hline
\end{tabular}

of these features as DIBs was not possible from our data. Certain very broad DIBs extending over wavelength regions which span two or more orders have been omitted due to the insufficient quality of order merging.

Table 2 shows a list of these suggested DIBs not included in the present survey. Seven of those diffuse interstellar bands denoted "certain" by Jenniskens \& Désert (1994) cannot be accurately measured in the present spectrum of $\mathrm{BD}+63^{\circ} 1964$. The $6741 \AA$ DIB lies between two orders and is therefore lost in our data. Another DIB at $6532 \AA$ spans two orders. We cannot exclude the possiblity of imperfect merging in this case due to the nearby strong 
Table 3. Possible additional Diffuse Interstellar Bands in $\mathrm{BD}+63^{\circ} 1964$. These DIBs have yet to be confirmed. Central wavelength, equivalent width $(E W)$ and full width at half maximum $(F W H M)$ are listed

\begin{tabular}{|crc|crc|}
\hline $\begin{array}{c}\lambda_{\text {DIB }} \\
(\AA)\end{array}$ & $\begin{array}{c}E W \\
(\AA)\end{array}$ & $\begin{array}{c}F W H M \\
(\AA)\end{array}$ & $\begin{array}{c}\lambda_{\text {DIB }} \\
(\AA)\end{array}$ & $\begin{array}{c}E W \\
(\AA)\end{array}$ & $\begin{array}{c}F W H M \\
(\AA)\end{array}$ \\
\hline 4968.87 & 65 & 6.54 & 5724.87 & 6 & 0.55 \\
4979.28 & 14 & 1.29 & 5806.52 & 15 & 1.54 \\
5098.84 & 5 & 0.48 & 5834.54 & 9 & 0.84 \\
5166.33 & 7 & 0.68 & 5966.11 & 8 & 0.84 \\
5167.28 & 8 & 0.69 & 6095.21 & 5 & 0.65 \\
5176.67 & 34 & 3.50 & 6102.48 & 12 & 1.77 \\
5428.63 & 12 & 1.11 & 6154.57 & 8 & 1.22 \\
5443.22 & 7 & 0.72 & 6293.29 & 5 & 0.41 \\
5525.48 & 8 & 0.74 & 6468.45 & 9 & 1.29 \\
5539.73 & 14 & 1.48 & 6476.81 & 5 & 0.55 \\
5559.93 & 6 & 1.06 & 6639.34 & 11 & 1.11 \\
5632.81 & 6 & 0.65 & 6808.36 & 7 & 0.56 \\
5634.73 & 7 & 0.89 & & & \\
\hline \multicolumn{7}{|c}{}
\end{tabular}

$\mathrm{H}-\alpha$ line. The absence of a third DIB, at $6451 \AA$, could possibly be due to its extension over two orders. This DIB however is not visible even when an optimal merging of the orders is obtained. The rest of the "certain" DIBs, except for one, are either too weak to be accurately measured or else absent from the spectrum. The remaining one, at $5850.2 \AA$, positively identified by Jenniskens et al. (1996), is absent because the resolution of the ELODIE spectrograph, at 42000 , is not sufficient to distinguish this very weak DIB from the stronger and broader band at $5849.8 \AA$. It was detected with the AURELIE spectrograph at Observatoire d'Haute Provence, France, which has a resolution in excess of 50000 (Jenniskens et al. 1996). Alternatively this is just a red wing of the $5849.8 \AA$ DIB. All the remaining DIBs not included in the survey have previously been classified as either probable or doubtful.

\subsection{Possible additional DIBs to be confirmed}

The spectrum of $\mathrm{BD}+63^{\circ} 1964$ provides us with a unique opportunity for finding and measuring new diffuse interstellar bands. The fact that previously known narrow DIBs are enhanced by a factor of $1.5-3$ toward this target lends credence to this. An absorption band has to fulfill certain criteria as mentioned in Sect. 5 before it can be considered as a new DIB.

The wealth of interstellar features in $\mathrm{BD}+63^{\circ} 1964$ 's spectrum has resulted in the discovery of 60 new "certain" DIBs based upon these criteria. There seems to be no doubt that this number is limited by the signal to noise of the present data and the magnitude $V=8.6$ for this exceptional target. However this seems one of the best targets for DIB surveys when studied with cross-dispersed echelle spectrographs on $2 \mathrm{~m}$ class telescopes. It is likely that an analysis of more spectra of higher quality and using a larger telescope would result in the positive detection of many more diffuse bands. In searching $\mathrm{BD}+63^{\circ}$ 1964's spectrum for new DIBs a number of weak absorption features which are probably of interstellar origin were listed. They did not fulfill the necessary criteria to be included as "certain" DIBs in this particular survey. In some cases the uncertainty was due to blended stellar lines or atmospheric lines, or stellar lines in HD 205021 at the wavelength corresponding to that of the suspect DIB coinciding with stellar lines in the synthetic stellar spectrum. In other cases it was difficult to distinguish the feature from localised random noise. Table 3 lists these possible DIBs.

The interest of the present survey comes from the exceptional quality of the spectrum of the line-of-sight of $\mathrm{BD}+63^{\circ} 1964$, with additional confirmation provided by spectra of the very reddened targets HD 183143 (a B7 star with a slow rotation) and $\mathrm{BD}+40^{\circ} 4220$ (a hot $\mathrm{O} 7$ star with a fast rotation). Due to the unusual enhancement of DIBs in $\mathrm{BD}+63^{\circ} 1964$ we could not confirm the existence of 25 possible DIBs in the other targets. To confirm or reject these possible DIBs one needs to observe higher signal-to-noise spectra of reddened stars. Those DIBs listed as possible could then be cross-checked with other stars.

\section{Statistics on the DIB population}

\subsection{DIB distribution vs wavelength}

In the previous section we discussed the identification of 226 diffuse interstellar bands in the line-of-sight of $\mathrm{BD}+63^{\circ} 1964$ with added confirmation in two other reddened reference targets and the possible detection of a number of others. Figures 2 and 4 show the distribution of both full widths and equivalent widths of all certain DIBs in the wavelength range of the survey. The blue end of the spectrum is characterised by both a lack of narrow DIBs and an increased abundance of broad DIBs. We rule out the possibility that this could be an instrumental bias. The lower sensitivity and increased abundance of stellar lines in the blue have already been discussed. The sensitivity limit for the survey can be estimated from the expression:

$\sigma_{E W}=\frac{\sqrt{2 \Delta \lambda F W H M}}{\frac{S}{N}}$

Assuming a Full Width at half maximum of typically $1 \AA$, for instance, we find that the sensitivity limit in the equivalent width is homogeneous from $4500 \AA$ to $6500 \AA$. Applying a $5 \sigma$ confidence level would lead to the detection of DIBs with an equivalent width $>12 \mathrm{~m} \AA$. The equivalent width of a DIB is wavelength-dependent,

$E W \propto N f \lambda^{2}$ 
where $N$ is the column density of the carrier and $f$ is the oscillator strength (Spitzer 1978). The DIBs in the red are obviously favoured. If constant column density and oscillator strength are assumed, a DIB with an equivalent width of $15 \mathrm{m \AA}$ at $6000 \AA$ would have an equivalent width of $9 \mathrm{~m} \AA$ at $4500 \AA$. This is however not sufficient to explain the lack of narrow DIBs we see in the blue. A physical explanation must be invoked to explain this phenomenon. Possible explanations might be that stronger and sharper fundamental transitions of large molecules occur in the red while secondary transitions in the blue are broadened due to limited lifetimes in excited levels of molecules (intercoupling between vibronic states).

We therefore find that the survey is homogeneously complete with narrow DIBs stronger than $15 \mathrm{~m} \AA$. The broader DIBs are not only concentrated towards shorter wavelengths. There is a second visible group of mediumbroad to broad DIBs around $6200 \AA$. The pattern of DIBs also seems to involve some clustering. Five clusters of narrow DIBs, four of which gather around one or more broader ones, can be seen above $5500 \AA$.

\subsection{Histograms of DIB widths and intensities}

Figures 3 and 5 show histograms depicting the spread of both full widths and equivalent widths of DIBs measured in $\mathrm{BD}+63^{\circ} 1964$. We can distinguish three populations of DIBs according to their $F W H M$. The most abundant are those whose widths lie between $0.6 \AA$ and $1.4 \AA$ (narrow DIBs). Medium-broad DIBs are seen between a width of $1.4 \AA$ and $3.2 \AA$, and broad DIBs, broader than $3.2 \AA$ do not show any dominant value in the histogram. It is clear that very broad DIBs are not completely surveyed, not only due to their difficulty of detection in echelle orders, but also due to their lower contrast (for a given Equivalent Width), their blend and mutual confusion with other features. However only very clearly defined DIBs would present an interest in terms of identification. The distribution of full widths at half maximum gives direct information on physical properties of the carriers (e.g. molecular rotational contours for narrow DIBs and lifetime broadening for some of the broad DIBs).

The corresponding plot of the equivalent width distribution on the contrary (considering the statistical dispersion) does not show any preferred equivalent width above the sensitivity cut-off. This is because the equivalent width is a product of three variables, the carrier column density, $N$, the transition oscillator strength, $f$, and $\lambda^{2}$, which take a wide and continuous range of values, with independent statistics.

\section{Conclusions}

\subsection{New detections and DIB statistics}

Figure 1 shows a composite spectrum of all DIBs seen toward the reddened targets in the wavelength range $3906-6812 \AA$. The detection of 60 new DIBs raised the total number of DIBs to 226 in this line-of-sight, along with 25 possible DIBs awaiting confirmation. A statistical analysis on the DIB population has clarified the presence of three main DIB groups based on their band widths and has illustrated the clustering of DIBs in selected wavelength bands.

\subsection{The enhancement of DIBs in $B D+63^{\circ} 1964 \mathrm{vs}$. other reference targets}

This survey of diffuse interstellar bands in the optical range has confirmed earlier reports of the unique nature of $\mathrm{BD}+63^{\circ} 1964$ compared to other heavily reddened targets, such as HD 183143 and $\mathrm{BD}+40^{\circ} 4220$ (Ehrenfreund et al. 1997). What mechanism could cause this unusual enhancement in DIB strengths and lead to the identification of many more hitherto undetected DIBs? The Ca II absorption lines at 3933 and $3968 \AA$, as well as the $\mathrm{CH}$ band at $4300 \AA$ seem to indicate at least two clouds of different velocities in the line-of-sight of $\mathrm{BD}+63^{\circ} 1964$. In order to understand the DIB behaviour, models of diffuse clouds have been performed using information on various atomic and molecular species residing in the line-of-sight toward $\mathrm{BD}+63^{\circ} 1964$. The models indicate that the lineof-sight toward this target passes through a cloud edge where ultra-violet radiation may trigger the ionization of DIB carriers (Tuairisg et al. 2000). Further modeling and observational efforts are required to constrain the environmental conditions in this exceptional line-of-sight.

\subsection{Future work}

Higher signal-to-noise spectra of $\mathrm{BD}+63^{\circ} 1964$ and other reddened targets would undoubtedly confirm many of the possible DIBs listed in Table 3, and possibly lead to the detection of more new DIBs. Important further work would be to complement this work with a comprehensive survey of DIBs in the near infra-red. Observations taken with the INT/MUSICOS spectrograph are currently under analysis. As previously mentioned, the ELODIE spectrograph has limitations for a survey of broader DIBs. Further observations of reddened targets using more suitable spectrographs to detect broad DIBs, are also desirable.

The new survey presented in this paper provides a new basis for comparison with spectroscopic measurements of DIB carrier candidates in the laboratory. However, with the large number of detected DIBs namely $\sim 200$ DIBs 
between 5400 and $6800 \AA$, we find on average one DIB every $6 \AA$. Therefore any identification in this range based only on wavelength coincidence could be obtained by pure chance and additional criteria for identification are necessary, such as multiple bands, band widths, DIB strength ratios and correlated variations in different environments (Ehrenfreund \& Foing 1995; Herbig 1995).

Follow-up studies of the surveyed DIBs by high resolution spectroscopy and the physical properties of the lineof-sight conditions will further help to reveal the nature of DIB carriers.

Acknowledgements. We thank the staff at OHP for their help during the observations. PE is a recipient of an APART fellowship. SÓT acknowledges support for a research stage at ESA Space Science Department.

\section{References}

Baranne A., Queloz D., Mayor M., et al., 1996, A\&AS 119, 373-390

Beals C.S., Blanchet G.H., 1937, PASP 49, 224

Cami J., Sonnentrucker P., Ehrenfreund P., Foing B.H., 1996, A\&A 326, 822

Chlewicki G., 1985, Observational constraints on multimodal Interstellar Grain Populations. Thesis Leiden University

Chlewicki G., Groot M.S., Van der Zwet G.P., Greenberg J.M., Alvarez P.P., Mampaso A., 1987, A\&A 173, 131

Ehrenfreund P., Foing B.H., 1995, Planetary Space Sci. 43, $10 / 11,1183$

Ehrenfreund P., Foing B.H., 1996, A\&A 307, L25

Ehrenfreund P., Cami J., Dartois E., Foing B.H., 1997, A\&A 318, L28

Ferlet R., Roueff E., Horani M., Rostas J., 1983, A\&A 125, L5
Ferlet R., Dennefeld M., 1984, A\&A 138, 303

Foing B.H., Ehrenfreund P., 1994, Nat 369, 296

Foing B.H., Ehrenfreund P., 1997, A\&A 317, L59

Freivogel P., Fulara J., Maier J.P., 1994, ApJ 431, L151-L154

Hayden-Schmitt W., Snow T.P., Jura M., Cochran W.D.S., 1981, ApJ 248, 128

Heger M.L., 1922, Lick Obs. Bull 10, 146

Herbig G.H., 1966, Zs. f. Astrophys. 64, 512

Herbig G.H., 1967, in: Radio Astronomy and the Galactic System, IAU Symp. 31, van Woerden H. (ed.), p. 85

Herbig G.H., 1975, ApJ 196, 129

Herbig G.H., 1988, ApJ 331, 999

Herbig G.H., 1995, ARA\&A 33, 19

Herbig G.H., Leka K.D., 1991, ApJ 382, 193

Hobbs L.W., 1984, ApJ 280, 132

Jenniskens P., Désert X., 1993, A\&A 274, 465

Jenniskens P., Désert X., 1994, A\&AS 106, 39

Jenniskens P., Porceddu I., Benvenuti P., Desert F.-X., 1996, A\&A 313, 649-656

Krelowski J., Sneden C., Hiltgen D., 1995, Planetary and Space Sci. 43, 1195-1203

Merrill P.W., 1934, PASP 46, 206

Salama F., Bakes E.L.O., Allamandola L.J., Tielens A.G.G.M., 1996, ApJ 458, 621

Sanner F., Snell R., Vanden Bout P., 1978, ApJ 226, 460

Sarre P.J., Miles J.R., Kerr T.H., Hibbins R.E, Fossey S.J., Somerville W.B., 1995, MNRAS 277, L41

Sorokin P., Glownia J.H., 1996, ApJ 473, 900

Spitzer L., 1978, Physical Processes in the Interstellar Medium. Wiley, New York

Tulej M., Kirkwood D.A., Pachkov M., Maier J.P., ApJ 506, L69-L73

Tuairisg S.Ó., Spaans M., Ehrenfreund P., Foing B.H., 2000 (in preparation for $\mathrm{A} \& \mathrm{~A}$ )

Ubachs W., Hinnen P.C., Reinhold E., 1997, ApJ 476, L93

Wilson R., 1958, ApJ 128, 57 
Table 4. Confirmed Diffuse Interstellar Bands measured towards BD $+63^{\circ}$ 1964, HD 183143 and BD $+40^{\circ} 4220$. The first Col. $(\lambda)$ gives the central absolute wavelength (in air) after correcting for the cloud velocity shift. $E W$ denotes the diffuse band equivalent width measured in the spectra of the reddened targets (tellurically corrected where necessary, as indicated in Fig. 6 through to Fig. 39). Errors for these values are denoted $\operatorname{Err}_{E W}$. FWHM denotes the full width at half maximum of the DIBs. Errors for these values are denoted $\operatorname{Err}_{F W H M}$. The final Col. (Ref.) cites the source of the first identification of the DIB in other targets. New DIBs are denoted by "New". Due to BD+63 1964 's $E_{(B-V)}$ of 1.01 normalisation to unit reddening is not necessary. DIBs which could not be measured due to stellar line contamination are marked with an asterisk $(*)$. A horizontal line denotes the absence of a DIB in the spectrum. Reference codes are: Hg22 - Heger (1922), Me34 - Merrill (1934), BB37 - Beals \& Blanchet (1937), Wi58 - Wilson (1958), He66 - Herbig (1966), He67 - Herbig (1967), He75 - Herbig (1975), Sa78 Sanner et al. (1978), HS81 - Hayden-Schmitt et al. (1991), F83 - Ferlet et al. (1983), FD84 - Ferlet \& Dennefeld (1984), Ho84 Hobbs (1984), Ch85 - Chlewicki (1985), Chl87 - Chlewicki (1987), He88 - Herbig (1988), HL91 - Herbig \& Leka (1991), JD93 Jenniskens \& Désert (1993), JD94 - Jenniskens \& Désert (1994), K95 - Krelowski et al. (1995), J96 - Jenniskens et al. (1996)

\begin{tabular}{|c|c|c|c|c|c|c|c|c|c|}
\hline $\begin{array}{l}\lambda \\
(\AA)\end{array}$ & $\begin{array}{c}E W \\
(\mathrm{~m} \AA) \\
\mathrm{BD}+63^{\circ}\end{array}$ & $\begin{array}{l}E r r_{E W} \\
\quad(\mathrm{~m} \AA) \\
1964\end{array}$ & $\begin{array}{c}E W \\
(\mathrm{~mA}) \\
\mathrm{HD} 1\end{array}$ & $\begin{array}{l}E r r_{E W} \\
\quad(\mathrm{~m} \AA) \\
143\end{array}$ & $\begin{array}{c}E W \\
(\mathrm{~m} \AA) \\
\mathrm{BD}+4\end{array}$ & $\begin{array}{l}E r r_{E W} \\
\quad(\mathrm{m \AA}) \\
4220\end{array}$ & $\begin{array}{c}F W H M \\
(\AA)\end{array}$ & $\begin{array}{c}E r r_{F W H M} \\
(\AA)\end{array}$ & Ref. \\
\hline 4175.46 & 314 & 81 & 450 & 95 & - & $\overline{ }$ & 17.2 & 5.22 & JD94 \\
\hline 4427.96 & 2760 & 120 & 2470 & 100 & 2236 & 250 & 17.5 & 1.24 & BB37 \\
\hline 4501.65 & 214 & 10 & 160 & 22 & 127 & 60 & 3.55 & 0.16 & He66 \\
\hline 4593.93 & 891 & 45 & 545 & 150 & 780 & 110 & 27.6 & 2.21 & JD94 \\
\hline 4666.65 & 125 & 7 & 54 & 10 & - & - & 6.61 & 0.38 & JD94 \\
\hline 4726.35 & 117 & 7 & 107 & 6 & 204 & 43 & 1.70 & 0.08 & He75 \\
\hline 4728.35 & 50 & 4 & 41 & 5 & 74 & 23 & 1.54 & 0.12 & He75 \\
\hline 4762.57 & 143 & 5 & 140 & 5 & 76 & 7 & 2.89 & 0.16 & He75 \\
\hline 4764.66 & 546 & 55 & 450 & 109 & 1020 & 250 & 29.0 & 3.56 & He75 \\
\hline 4780.10 & 55 & 4 & 53 & 3 & 59 & 5 & 1.74 & 0.22 & He75 \\
\hline 4825.94 & 347 & 27 & 377 & 65 & - & - & 20.4 & 2.17 & JD94 \\
\hline 4882.56 & 466 & 20 & 950 & 60 & 1900 & 350 & 14.9 & 0.76 & Wi58 \\
\hline 4951.05 & 8 & 2 & 6 & 2 & 5 & 2 & 0.67 & 0.11 & New \\
\hline 4963.03 & 218 & 12 & 239 & 110 & 540 & 200 & 21.3 & 2.70 & JD94 \\
\hline 4963.89 & 31 & 3 & 24 & 2 & 54 & 4 & 0.71 & 0.08 & JD94 \\
\hline 4984.78 & 19 & 2 & 8 & 3 & 14 & 3 & 0.67 & 0.16 & JD94 \\
\hline 5038.59 & 344 & 33 & 421 & 105 & 541 & 150 & 26.9 & 2.50 & JD94 \\
\hline 5061.56 & 9 & 2 & 10 & 2 & 10 & 5 & 0.64 & 0.15 & New \\
\hline 5110.81 & 30 & 6 & 45 & 10 & 35 & 16 & 2.45 & 0.51 & JD94 \\
\hline 5170.69 & 18 & 3 & $*$ & $*$ & 10 & 50 & 0.87 & 0.18 & New \\
\hline 5176.00 & 9 & 2 & 11 & 2 & 13 & 4 & 0.76 & 0.20 & New \\
\hline 5236.34 & 49 & 3 & 51 & 23 & 34 & 7 & 1.95 & 0.10 & New \\
\hline 5247.91 & 6 & 3 & 6 & 2 & 22 & 8 & 0.39 & 0.20 & New \\
\hline 5349.16 & 10 & 3 & 8 & 2 & 15 & 3 & 1.16 & 0.47 & New \\
\hline 5359.58 & 41 & 9 & 52 & 11 & 55 & 10 & 3.92 & 0.77 & He67 \\
\hline 5363.78 & 76 & 7 & 124 & 10 & 130 & 20 & 2.54 & 0.24 & JD94 \\
\hline 5370.36 & 29 & 4 & 11 & 3 & 15 & 5 & 3.25 & 0.61 & New \\
\hline 5404.52 & 33 & 2 & 41 & 2 & 30 & 4 & 1.06 & 0.08 & $\mathrm{He} 75$ \\
\hline 5418.39 & 109 & 9 & 89 & 12 & - & - & 8.85 & 0.39 & $\mathrm{He} 75$ \\
\hline 5419.05 & 20 & 2 & 12 & 3 & 25 & 5 & 1.07 & 0.11 & New \\
\hline 5449.76 & 290 & 12 & 345 & 34 & 242 & 40 & 9.53 & 0.50 & $\mathrm{He} 75$ \\
\hline 5450.95 & 696 & 22 & 444 & 150 & 619 & 100 & 31.2 & 1.46 & JD94 \\
\hline 5487.54 & 82 & 8 & 67 & 4 & 111 & 20 & 2.51 & 0.32 & $\mathrm{He} 75$ \\
\hline 5487.90 & 166 & 12 & 117 & 15 & 190 & 50 & 6.67 & 0.54 & JD94 \\
\hline
\end{tabular}


Table 4. continued

\begin{tabular}{|c|c|c|c|c|c|c|c|c|c|}
\hline $\begin{array}{c}\lambda \\
(\AA)\end{array}$ & $\begin{array}{c}E W \\
(\mathrm{~m} \AA) \\
\mathrm{BD}+63^{\circ}\end{array}$ & $\begin{array}{c}E r r_{E W} \\
(\mathrm{~m} \AA) \\
1964\end{array}$ & $\begin{array}{c}E W \\
(\mathrm{~mA}) \\
\mathrm{HD}\end{array}$ & $\begin{array}{r}E r r_{E W} \\
\quad(\mathrm{~m} \AA) \\
143\end{array}$ & $\begin{array}{c}E W \\
(\mathrm{~m} \AA) \\
\mathrm{BD}+4\end{array}$ & $\begin{array}{r}E r r_{E W} \\
\quad(\mathrm{~m} \AA) \\
4220\end{array}$ & $\begin{array}{c}F W H M \\
(\AA)\end{array}$ & $\begin{array}{c}E r r_{F W H M} \\
(\AA)\end{array}$ & Ref. \\
\hline 5490.52 & 8 & 2 & 7 & 2 & 7 & 3 & 0.75 & 0.19 & New \\
\hline 5494.10 & 51 & 3 & 30 & 2 & 27 & 3 & 0.86 & 0.07 & He75 \\
\hline 5497.00 & 22 & 5 & 17 & 4 & 17 & 5 & 2.17 & 0.90 & New \\
\hline 5503.16 & 5 & 2 & 8 & 2 & $<14$ & 7 & 0.42 & 0.15 & New \\
\hline 5506.06 & 7 & 2 & $*$ & $*$ & 11 & 3 & 0.58 & 0.24 & New \\
\hline 5508.03 & 103 & 6 & 121 & 16 & 108 & 11 & 2.77 & 0.36 & He75 \\
\hline 5512.66 & 19 & 2 & 8 & 2 & 15 & 3 & 0.69 & 0.07 & New \\
\hline 5515.97 & 8 & 3 & 7 & 2 & 8 & 3 & 1.06 & 0.35 & New \\
\hline 5524.47 & 11 & 2 & $<8$ & 4 & 13 & 4 & 0.91 & 0.24 & JD94 \\
\hline 5535.26 & 28 & 4 & 40 & 4 & 42 & 5 & 1.35 & 0.13 & JD94 \\
\hline 5537.51 & 95 & 18 & 85 & 11 & 100 & 20 & 5.42 & 0.29 & He75 \\
\hline 5541.78 & 13 & 3 & 5 & 2 & 11 & 2 & 0.68 & 0.21 & JD94 \\
\hline 5545.02 & 38 & 4 & 30 & 2 & 27 & 3 & 0.85 & 0.09 & He75 \\
\hline 5546.52 & 11 & 3 & $<8$ & 4 & 7 & 2 & 0.68 & 0.17 & New \\
\hline 5556.27 & 9 & 3 & 12 & 2 & 7 & 3 & 0.90 & 0.31 & New \\
\hline 5563.06 & 7 & 3 & $<8$ & 4 & 8 & 3 & 0.85 & 0.30 & New \\
\hline 5569.08 & 6 & 2 & 7 & 2 & 9 & 3 & 0.35 & 0.16 & New \\
\hline 5594.54 & 16 & 3 & 10 & 2 & 16 & 3 & 0.86 & 0.15 & New \\
\hline 5597.38 & 16 & 4 & 5 & 2 & 8 & 4 & 2.59 & 1.01 & New \\
\hline 5600.49 & 9 & 2 & 9 & 2 & 7 & 3 & 1.17 & 0.39 & New \\
\hline 5609.96 & 33 & 6 & 23 & 3 & 50 & 7 & 2.14 & 0.57 & JD94 \\
\hline 5645.43 & 11 & 2 & * & $*$ & 16 & 3 & 0.71 & 0.15 & New \\
\hline 5705.10 & 166 & 10 & 163 & 10 & 214 & 24 & 2.75 & 0.12 & He67 \\
\hline 5709.40 & 49 & 9 & 29 & 3 & 43 & 5 & 1.71 & 0.27 & JD94 \\
\hline 5719.68 & 31 & 4 & 25 & 3 & 24 & 3 & 1.04 & 0.20 & JD94 \\
\hline 5746.21 & 52 & 4 & 48 & 5 & 35 & 4 & 2.84 & 0.20 & JD94 \\
\hline 5760.64 & 5 & 3 & $<8$ & 4 & 6 & 2 & 0.61 & 0.13 & K95 \\
\hline 5762.80 & 16 & 3 & 8 & 2 & $<12$ & 6 & 0.95 & 0.11 & JD93 \\
\hline 5766.17 & 41 & 3 & 21 & 3 & 29 & 4 & 1.08 & 0.07 & Ch87 \\
\hline 5769.32 & 15 & 2 & 12 & 3 & 18 & 3 & 1.26 & 0.28 & JD93 \\
\hline 5772.67 & 32 & 3 & 24 & 3 & 44 & 5 & 1.45 & 0.14 & JD93 \\
\hline 5776.13 & 420 & 31 & 640 & 39 & 501 & 32 & 15.2 & 2.20 & Hg22 \\
\hline 5776.21 & 28 & 4 & 19 & 4 & 29 & 4 & 2.00 & 0.30 & JD93 \\
\hline 5780.55 & 699 & 4 & 770 & 8 & 713 & 11 & 2.22 & 0.01 & Hg22 \\
\hline 5784.86 & 9 & 2 & 11 & 2 & 15 & 5 & 0.92 & 0.36 & JD93 \\
\hline 5789.04 & 15 & 3 & 20 & 2 & 52 & 6 & 1.16 & 0.15 & JD93 \\
\hline 5793.13 & 11 & 2 & 5 & 2 & 9 & 3 & 0.95 & 0.19 & K95 \\
\hline 5796.07 & 155 & 16 & 138 & 18 & 236 & 30 & 6.65 & 0.70 & He67 \\
\hline 5797.08 & 258 & 15 & 187 & 10 & 179 & 7 & 0.96 & 0.05 & $\mathrm{Hg} 22$ \\
\hline 5809.53 & 59 & 7 & 26 & 3 & $*$ & * & 1.83 & 0.25 & JD94 \\
\hline 5810.21 & 25 & 7 & 10 & 2 & $*$ & $*$ & 1.74 & 0.30 & JD93 \\
\hline 5815.80 & 4 & 2 & $<8$ & 4 & 8 & 3 & 0.65 & 0.27 & JD93 \\
\hline 5818.47 & 8 & 3 & 22 & 2 & 13 & 3 & 0.81 & 0.32 & JD93 \\
\hline 5828.56 & 15 & 2 & 12 & 2 & 22 & 3 & 0.87 & 0.16 & JD93 \\
\hline 5837.92 & 6 & 2 & 18 & 2 & 6 & 3 & 0.52 & 0.28 & J96 \\
\hline 5840.62 & 9 & 3 & $<8$ & 4 & $<8$ & 4 & 0.64 & 0.23 & J96 \\
\hline 5842.38 & 6 & 2 & $<4$ & 4 & $<4$ & 4 & 0.45 & 0.20 & J96 \\
\hline 5843.30 & 5 & 3 & $<8$ & 4 & $<8$ & 4 & 0.36 & 0.10 & J96 \\
\hline 5843.69 & 4 & 3 & $<8$ & 4 & $<8$ & 4 & 0.31 & 0.15 & J96 \\
\hline 5844.28 & 87 & 11 & 132 & 12 & 111 & 14 & 4.61 & 0.59 & He67 \\
\hline 5844.95 & 10 & 3 & 12 & 2 & $<8$ & 4 & 0.65 & 0.21 & JD94 \\
\hline 5849.81 & 119 & 2 & 62 & 4 & 84 & 4 & 1.07 & 0.01 & He67 \\
\hline
\end{tabular}


Table 4. continued

\begin{tabular}{|c|c|c|c|c|c|c|c|c|c|}
\hline \multirow[t]{2}{*}{$\begin{array}{c}\lambda \\
(\AA)\end{array}$} & $\begin{array}{c}E W \\
(\mathrm{~m} \AA)\end{array}$ & $\begin{array}{r}E r r_{E W} \\
(\mathrm{~m} \AA)\end{array}$ & $\begin{array}{c}E W \\
(\mathrm{~m} \AA)\end{array}$ & $\begin{array}{r}E r r_{E W} \\
(\mathrm{~m} \AA)\end{array}$ & $\begin{array}{c}E W \\
(\mathrm{~m} \AA)\end{array}$ & $\begin{array}{r}E r r_{E W} \\
(\mathrm{~m} \AA)\end{array}$ & \multirow[t]{2}{*}{$\begin{array}{c}F W H M \\
(\AA)\end{array}$} & \multirow[t]{2}{*}{$\begin{array}{c}\operatorname{Err}_{F W H M} \\
(\AA)\end{array}$} & \multirow[t]{2}{*}{ Ref. } \\
\hline & \multicolumn{4}{|c|}{$\mathrm{BD}+63^{\circ} 1964$} & \multicolumn{2}{|c|}{$\mathrm{BD}+40^{\circ} 4220$} & & & \\
\hline 5854.85 & 17 & 5 & 19 & 3 & 15 & 4 & 2.13 & 0.67 & JD94 \\
\hline 5900.58 & 12 & 3 & 12 & 2 & 16 & 3 & 0.75 & 0.16 & K95 \\
\hline 5910.54 & 23 & 3 & $<14$ & 7 & 23 & 4 & 0.89 & 0.09 & K95 \\
\hline 5923.51 & 34 & 3 & 23 & 3 & 33 & 3 & 0.89 & 0.16 & K95 \\
\hline 5925.81 & 20 & 3 & 19 & 2 & 17 & 3 & 0.90 & 0.13 & K95 \\
\hline 5945.53 & 10 & 2 & 10 & 2 & 15 & 5 & 0.72 & 0.24 & K95 \\
\hline 5947.29 & 35 & 3 & 16 & 2 & 19 & 3 & 0.95 & 0.09 & K95 \\
\hline 5948.87 & 10 & 3 & 6 & 2 & $<8$ & 4 & 0.71 & 0.20 & K95 \\
\hline 5958.89 & 32 & 3 & 52 & 7 & 30 & 4 & 1.28 & 0.08 & New \\
\hline 5973.78 & 10 & 2 & 8 & 2 & 16 & 4 & 0.74 & 0.13 & New \\
\hline 5975.66 & 9 & 2 & $<8$ & 4 & 5 & 2 & 0.49 & 0.08 & K95 \\
\hline 5982.77 & 17 & 3 & 16 & 2 & 7 & 3 & 1.23 & 0.18 & $\mathrm{He} 75$ \\
\hline 5986.60 & 13 & 3 & 6 & 2 & $<8$ & 4 & 1.30 & 0.29 & K95 \\
\hline 5988.04 & 12 & 3 & 12 & 2 & 5 & 2 & 0.91 & 0.13 & K95 \\
\hline 5989.51 & 7 & 2 & 6 & 2 & 5 & 2 & 0.95 & 0.24 & K95 \\
\hline 5995.73 & 9 & 4 & 7 & 2 & 7 & 3 & 0.84 & 0.31 & K95 \\
\hline 6004.80 & 36 & 3 & 30 & 3 & 15 & 3 & 2.92 & 0.38 & HL91 \\
\hline 6010.48 & 88 & 4 & 105 & 7 & 129 & 7 & 2.90 & 0.15 & He75 \\
\hline 6019.45 & 9 & 2 & 17 & 2 & 24 & 3 & 0.58 & 0.11 & JD94 \\
\hline 6027.09 & 37 & 4 & 35 & 4 & 45 & 4 & 2.28 & 0.25 & JD94 \\
\hline 6037.47 & 39 & 3 & 33 & 4 & 78 & 8 & 2.26 & 0.19 & JD94 \\
\hline 6042.84 & 296 & 32 & 262 & 23 & 367 & 40 & 14.3 & 3.33 & He75 \\
\hline 6059.88 & 15 & 5 & 9 & 2 & 20 & 4 & 1.20 & 0.50 & JD94 \\
\hline 6061.52 & 8 & 4 & 7 & 2 & $<8$ & 4 & 0.86 & 0.34 & New \\
\hline 6065.19 & 15 & 3 & 15 & 2 & 15 & 3 & 0.90 & 0.17 & JD94 \\
\hline 6068.45 & 8 & 3 & 6 & 2 & 10 & 3 & 1.34 & 0.25 & New \\
\hline 6071.08 & 16 & 5 & 16 & 3 & 52 & 20 & 2.29 & 0.98 & JD94 \\
\hline 6084.94 & 9 & 2 & 10 & 3 & 10 & 2 & 1.13 & 0.36 & New \\
\hline 6089.79 & 43 & 2 & 24 & 3 & 23 & 3 & 0.68 & 0.03 & HL91 \\
\hline 6108.14 & 10 & 2 & 11 & 2 & 10 & 3 & 0.60 & 0.15 & JD94 \\
\hline 6113.20 & 44 & 3 & 43 & 3 & 25 & 3 & 1.02 & 0.04 & He75 \\
\hline 6116.74 & 13 & 2 & 14 & 2 & 15 & 2 & 0.86 & 0.08 & JD94 \\
\hline 6118.66 & 5 & 2 & 5 & 2 & 8 & 3 & 0.43 & 0.10 & New \\
\hline 6139.94 & 18 & 2 & 23 & 3 & 22 & 3 & 0.64 & 0.03 & JD94 \\
\hline 6145.41 & 9 & 9 & $<8$ & 4 & $<12$ & 6 & 0.96 & 0.25 & New \\
\hline 6147.02 & 5 & 2 & * & $*$ & 15 & 4 & 0.90 & 0.32 & New \\
\hline 6151.15 & 15 & 2 & $*$ & $*$ & 10 & 3 & 1.44 & 0.20 & New \\
\hline 6161.83 & 11 & 2 & 8 & 2 & 11 & 2 & 0.91 & 0.13 & New \\
\hline 6165.72 & 15 & 3 & 10 & 2 & 7 & 2 & 1.56 & 0.23 & New \\
\hline 6170.71 & 15 & 3 & 17 & 3 & 9 & 3 & 1.31 & 0.17 & New \\
\hline 6173.65 & 907 & 22 & 1059 & 120 & 1075 & 81 & 23.1 & 1.24 & Wi58 \\
\hline 6177.72 & 14 & 3 & 8 & 2 & 10 & 3 & 1.91 & 0.48 & New \\
\hline 6185.89 & 15 & 3 & 9 & 2 & 11 & 3 & 0.93 & 0.15 & New \\
\hline 6187.19 & 19 & 3 & 10 & 2 & 10 & 3 & 1.24 & 0.22 & New \\
\hline 6189.31 & 14 & 3 & 7 & 2 & 15 & 3 & 1.27 & 0.20 & JD94 \\
\hline 6194.57 & 5 & 2 & 6 & 2 & 6 & 3 & 0.31 & 0.08 & HS81 \\
\hline 6194.98 & 5 & 2 & 5 & 2 & 7 & 2 & 0.28 & 0.04 & JD94 \\
\hline 6195.99 & 79 & 3 & 86 & 5 & 66 & 3 & 0.68 & 0.03 & He67 \\
\hline 6198.82 & 6 & 3 & 11 & 3 & $<18$ & 9 & 0.68 & 0.23 & JD94 \\
\hline 6203.06 & 139 & 13 & 125 & 18 & 124 & 22 & 1.30 & 0.03 & He67 \\
\hline 6204.22 & 135 & 9 & 123 & 9 & 143 & 20 & 4.92 & 0.51 & He67 \\
\hline 6207.52 & 132 & 17 & 160 & 17 & 150 & 30 & 9.34 & 1.47 & JD94 \\
\hline 6211.74 & 22 & 2 & 9 & 2 & 14 & 3 & 1.27 & 0.17 & K95 \\
\hline
\end{tabular}


Table 4. continued

\begin{tabular}{|c|c|c|c|c|c|c|c|c|c|}
\hline $\begin{array}{c}\lambda \\
(\AA)\end{array}$ & $\begin{array}{l}E W \\
(\mathrm{~m} \AA) \\
\mathrm{BD}+63^{\circ}\end{array}$ & $\begin{array}{c}E r r_{E W} \\
(\mathrm{~m} \AA) \\
1964\end{array}$ & $\begin{array}{c}E W \\
(\mathrm{~m} \AA) \\
\mathrm{HD}\end{array}$ & $\begin{array}{l}E r r_{E W} \\
\quad(\mathrm{~m} \AA) \\
143\end{array}$ & $\begin{array}{c}E W \\
(\mathrm{~m} \AA) \\
\mathrm{BD}+4\end{array}$ & $\begin{array}{l}\operatorname{Err}_{E W} \\
\quad(\mathrm{m \AA}) \\
4220\end{array}$ & $\begin{array}{c}F W H M \\
(\AA)\end{array}$ & $\begin{array}{c}E r r_{F W H M} \\
(\AA)\end{array}$ & Ref. \\
\hline 6212.95 & 4 & 2 & 5 & 2 & 10 & 3 & 0.52 & 0.19 & Ch85 \\
\hline 6215.80 & 20 & 3 & 13 & 2 & 22 & 4 & 1.84 & 0.44 & JD94 \\
\hline 6220.86 & 11 & 3 & 7 & 3 & 11 & 4 & 0.84 & 0.27 & K95 \\
\hline 6223.53 & 6 & 2 & 10 & 2 & 5 & 2 & 0.59 & 0.18 & JD94 \\
\hline 6226.02 & 11 & 2 & 6 & 2 & 11 & 3 & 0.82 & 0.12 & K95 \\
\hline 6234.01 & 28 & 4 & 18 & 3 & 12 & 2 & 0.80 & 0.15 & He75 \\
\hline 6236.71 & 10 & 4 & 9 & 2 & 9 & 3 & 1.07 & 0.42 & JD93 \\
\hline 6244.42 & 13 & 4 & 22 & 3 & 6 & 2 & 0.92 & 0.12 & New \\
\hline 6245.36 & 28 & 3 & 22 & 4 & 20 & 7 & 2.22 & 0.71 & K95 \\
\hline 6250.77 & 16 & 3 & 8 & 3 & 10 & 3 & 1.40 & 0.32 & New \\
\hline 6269.82 & 84 & 5 & 115 & 10 & 100 & 9 & 1.22 & 0.10 & He67 \\
\hline 6270.45 & 86 & 11 & 130 & 11 & 128 & 17 & 4.34 & 0.91 & He75 \\
\hline 6278.17 & 10 & 5 & 32 & 5 & 28 & 5 & 1.16 & 0.57 & JD93 \\
\hline 6280.48 & 15 & 5 & 10 & 2 & 17 & 4 & 1.03 & 0.37 & JD93 \\
\hline 6283.30 & 899 & 45 & 1240 & 102 & 1172 & 120 & 10.1 & 0.12 & JD93 \\
\hline 6284.09 & 540 & 11 & 689 & 19 & 712 & 20 & 2.71 & 0.06 & Me34 \\
\hline 6287.57 & 13 & 4 & 12 & 2 & 7 & 2 & 0.52 & 0.24 & JD93 \\
\hline 6289.70 & 21 & 6 & 20 & 3 & 18 & 5 & 1.58 & 1.00 & JD93 \\
\hline 6302.29 & 31 & 3 & 20 & 3 & 30 & 4 & 3.19 & 0.39 & New \\
\hline 6308.92 & 47 & 3 & 78 & 10 & 90 & 20 & 2.08 & 0.09 & HL91 \\
\hline 6311.53 & 268 & 32 & 390 & 48 & 230 & 120 & 23.0 & 2.64 & He75 \\
\hline 6317.75 & 75 & 11 & 60 & 7 & 139 & 22 & 2.97 & 0.19 & JD93 \\
\hline 6324.81 & 22 & 6 & 22 & 2 & 17 & 3 & 1.20 & 0.26 & JD93 \\
\hline 6330.14 & 16 & 2 & 18 & 3 & 28 & 10 & 0.98 & 0.15 & JD93 \\
\hline 6353.18 & 46 & 3 & 38 & 3 & 30 & 3 & 2.09 & 0.12 & He75 \\
\hline 6358.54 & 20 & 3 & 15 & 2 & 18 & 3 & 1.88 & 0.22 & New \\
\hline 6360.21 & 834 & 55 & 920 & 200 & 830 & 125 & 30.0 & 2.50 & HL91 \\
\hline 6362.23 & 31 & 4 & 32 & 2 & 29 & 6 & 1.66 & 0.21 & HL91 \\
\hline 6367.28 & 18 & 2 & 18 & 2 & 12 & 3 & 0.67 & 0.09 & Ho84 \\
\hline 6371.92 & 6 & 3 & $*$ & $*$ & 11 & 2 & 0.74 & 0.29 & K95 \\
\hline 6376.02 & 27 & 4 & 44 & 4 & 38 & 4 & 0.64 & 0.09 & He75 \\
\hline 6376.57 & 52 & 7 & 20 & 4 & 54 & 6 & 1.80 & 0.29 & JD94 \\
\hline 6379.22 & 176 & 3 & 107 & 3 & 85 & 4 & 0.81 & 0.01 & He75 \\
\hline 6396.95 & 39 & 3 & 55 & 4 & 37 & 5 & 1.35 & 0.12 & HL91 \\
\hline 6400.37 & 18 & 2 & 12 & 2 & 6 & 3 & 1.10 & 0.11 & K95 \\
\hline 6410.08 & 6 & 2 & $<8$ & 4 & 7 & 3 & 0.45 & 0.14 & New \\
\hline 6414.15 & 5 & 3 & 22 & 2 & 6 & 3 & 0.90 & 0.25 & HL91 \\
\hline 6414.76 & 89 & 9 & 130 & 22 & 174 & 25 & 8.20 & 1.05 & HL91 \\
\hline 6417.27 & 5 & 2 & 8 & 2 & $<14$ & 7 & 0.61 & 0.19 & New \\
\hline 6418.54 & 5 & 2 & $<8$ & 4 & 5 & 2 & 0.71 & 0.18 & New \\
\hline 6425.61 & 26 & 3 & 21 & 2 & 32 & 3 & 0.93 & 0.10 & He67 \\
\hline 6439.42 & 35 & 3 & 39 & 3 & 22 & 3 & 1.25 & 0.10 & HL91 \\
\hline 6445.25 & 39 & 4 & 42 & 3 & 34 & 3 & 0.83 & 0.11 & HL91 \\
\hline 6449.30 & 32 & 3 & 21 & 2 & 14 & 3 & 1.16 & 0.11 & HL91 \\
\hline 6456.08 & 47 & 3 & 49 & 7 & 62 & 7 & 1.29 & 0.08 & New \\
\hline 6460.31 & 13 & 3 & 10 & 2 & $<14$ & 7 & 0.73 & 0.34 & JD94 \\
\hline 6463.63 & 30 & 2 & 23 & 3 & 35 & 5 & 1.06 & 0.07 & New \\
\hline 6466.95 & 15 & 2 & 12 & 2 & 7 & 3 & 0.79 & 0.06 & New \\
\hline 6480.48 & 8 & 2 & $<8$ & 4 & 28 & 11 & 0.54 & 0.08 & New \\
\hline 6489.29 & 12 & 3 & 6 & 2 & $<8$ & 4 & 0.64 & 0.08 & New \\
\hline 6492.17 & 11 & 2 & 20 & 2 & 17 & 3 & 0.78 & 0.15 & JD94 \\
\hline 6492.92 & 155 & 21 & 135 & 24 & 180 & 30 & 9.67 & 1.17 & JD94 \\
\hline 6497.79 & 6 & 3 & 4 & 2 & 11 & 2 & 0.73 & 0.33 & JD94 \\
\hline 6520.95 & 35 & 3 & 41 & 3 & 54 & 7 & 1.35 & 0.10 & JD94 \\
\hline
\end{tabular}


Table 4. continued

\begin{tabular}{|c|c|c|c|c|c|c|c|c|c|}
\hline \multirow[t]{2}{*}{$\begin{array}{c}\lambda \\
(\AA)\end{array}$} & $\begin{array}{c}E W \\
(\mathrm{~m} \AA)\end{array}$ & $\begin{array}{r}E r r_{E W} \\
(\mathrm{~m} \AA)\end{array}$ & $\begin{array}{c}E W \\
(\mathrm{~m} \AA)\end{array}$ & $\begin{array}{r}E r r_{E W} \\
(\mathrm{~m} \AA)\end{array}$ & $\begin{array}{c}E W \\
(\mathrm{~m} \AA)\end{array}$ & $\begin{array}{r}\operatorname{Err}_{E W} \\
(\mathrm{~m} \AA)\end{array}$ & \multirow[t]{2}{*}{$\begin{array}{c}F W H M \\
(\AA)\end{array}$} & \multirow[t]{2}{*}{$\begin{array}{c}\operatorname{Err}_{F W H M} \\
(\AA)\end{array}$} & \multirow[t]{2}{*}{ Ref. } \\
\hline & \multicolumn{4}{|c|}{$\mathrm{BD}+63^{\circ} 1964$} & \multicolumn{2}{|c|}{$\mathrm{BD}+40^{\circ} 4220$} & & & \\
\hline 6536.86 & 6 & 3 & 8 & 2 & 22 & 4 & 0.69 & 0.30 & $\begin{array}{l}\text { JD94 } \\
\end{array}$ \\
\hline 6553.76 & 23 & 2 & 17 & 3 & 17 & 3 & 0.79 & 0.05 & New \\
\hline 6591.03 & 101 & 17 & 97 & 4 & - & - & 4.19 & 0.46 & JD94 \\
\hline 6597.47 & 13 & 3 & 20 & 3 & 15 & 3 & 0.79 & 0.21 & He75 \\
\hline 6613.63 & 314 & 4 & 320 & 4 & 300 & 5 & 1.14 & 0.01 & Me34 \\
\hline 6622.59 & 20 & 4 & 18 & 3 & 14 & 3 & 2.07 & 0.64 & K95 \\
\hline 6632.86 & 13 & 3 & 22 & 3 & 20 & 3 & 0.95 & 0.29 & JD94 \\
\hline 6635.50 & 7 & 2 & 7 & 2 & 10 & 2 & 0.63 & 0.20 & K95 \\
\hline 6644.33 & 15 & 3 & 29 & 3 & 25 & 5 & 1.43 & 0.26 & New \\
\hline 6645.95 & 8 & 2 & 11 & 2 & 9 & 3 & 0.58 & 0.20 & K95 \\
\hline 6660.62 & 57 & 3 & 75 & 4 & 51 & 3 & 0.82 & 0.02 & JD94 \\
\hline 6662.25 & 5 & 2 & 8 & 2 & 35 & 4 & 0.43 & 0.12 & New \\
\hline 6681.07 & 27 & 4 & $*$ & $*$ & * & $*$ & 2.01 & 0.18 & K95 \\
\hline 6684.83 & 8 & 3 & 5 & 2 & * & * & 1.09 & 0.19 & New \\
\hline 6686.46 & 5 & 2 & $<8$ & 4 & $*$ & $*$ & 0.63 & 0.13 & New \\
\hline 6689.38 & 10 & 3 & 17 & 2 & 22 & 2 & 0.61 & 0.17 & K95 \\
\hline 6694.40 & 11 & 2 & 8 & 2 & 10 & 2 & 1.19 & 0.33 & JD94 \\
\hline 6699.28 & 41 & 3 & 48 & 2 & 27 & 3 & 0.99 & 0.07 & Sa78 \\
\hline 6701.87 & 15 & 3 & 20 & 2 & 7 & 2 & 0.83 & 0.13 & $\mathrm{Fe} 83$ \\
\hline 6707.73 & 13 & 3 & $<8$ & 4 & 6 & 2 & 1.38 & 0.41 & New \\
\hline 6709.65 & 16 & 4 & 13 & 2 & * & $*$ & 1.76 & 0.44 & FD84 \\
\hline 6719.58 & 20 & 3 & * & $*$ & 11 & 3 & 3.05 & 0.20 & New \\
\hline 6729.20 & 17 & 2 & 13 & 2 & 14 & 4 & 0.81 & 0.09 & New \\
\hline 6733.35 & 16 & 2 & 26 & 3 & $<8$ & 4 & 1.28 & 0.17 & New \\
\hline 6765.39 & 11 & 3 & 12 & 2 & 7 & 2 & 0.86 & 0.19 & New \\
\hline 6770.23 & 9 & 2 & 18 & 2 & 10 & 2 & 0.58 & 0.12 & He75 \\
\hline 6789.64 & 15 & 3 & 9 & 2 & 10 & 2 & 2.54 & 0.38 & He88 \\
\hline 6792.54 & 11 & 3 & 19 & 3 & 9 & 2 & 0.66 & 0.14 & He88 \\
\hline 6795.18 & 11 & 4 & 15 & 2 & 5 & 2 & 0.92 & 0.33 & He88 \\
\hline 6801.39 & 7 & 3 & 15 & 2 & 12 & 3 & 0.95 & 0.34 & He88 \\
\hline 6804.82 & 13 & 3 & 7 & 2 & 13 & 3 & 1.86 & 0.27 & He88 \\
\hline 6811.30 & 14 & 3 & 18 & 3 & 21 & 4 & 0.95 & 0.23 & He88 \\
\hline 6812.39 & 12 & 3 & 20 & 3 & 12 & 3 & 1.03 & 0.33 & He88 \\
\hline
\end{tabular}

